\title{
Publication by junior doctors: why do they do it?
}

\author{
Richard Duffett
}

\begin{abstract}
This paper examines the experience and moltuation of junior doctors who successtully published in the Psychlatilic Bulletin and the Brtitish Joumal of Psychlatry, over a four month period. Most articles took over a year from first involvement to successful publication, although this varied according to the type of article. While having published is recognised to increase the likelihood of appointment to registrar and senior registrar posts, interest in the subject is frequently clted as a more important reason to publish among authors than enhancement of their curriculum vitae.
\end{abstract}

Publication and research by junior doctors (JD) has been the subject of many articles in the Psychiatric Bulletin. Lewis (1991) found that candidates for senior registrar (SR) posts were more likely to be shortlisted if they had a 'publication'. Katona \& Robertson (1993) replicated this and found that publication, particularly of datacontaining' articles, predicted appointments to SR posts and short-listing for registrar (reg) posts. Research experience without publication, however, did not increase the likelihood of either short-listing or appointment. While appointment panels may look for other qualities and experlences in candidates, and not necessarily consider publication essential (Izaguirre, 1993), the fact remains that applicants who have published are far more likely to be successful.

Publication has mainly been considered as a single entity, but may vary from a brief letter giving an opinion on a post (a non-datacontaining article), to the product of many years research. This study aims at examining the experience and motivation of JDs (of SHO, reg and SR grades) who publish in the Psychiatric Bulletin (PB) and British Journal of Psychiatry (BJP).

\section{The study}

Over a four month period all JDs who published articles, excluding letters (as grade is not specified) in PB or BJP were identified. Journal, position of authorship and training grade were recorded. Articles were identified a priori as audit articles (measuring current or recently changed practice), research (designed to test a hypoth- esis), surveys (canvassing opinions e.g. from colleagues), literature reviews, case reports, or 'non-data-containing' (e.g. opinions, conference briefings, foreign reports etc.). Questionnaires regarding the index article were sent to all identified authors and included questions on gender, time from first involvement to publication, involvement in the different stages to publication, time when the work was carried out, supervision, motivation and previous publications.

\section{Findings}

Seventy-seven authors were identified, 53 in the PB (35 first or sole authors) and 24 in the B.JP ( 12 first or sole authors). Forty-seven were SRs, 29 regs and one an SHO (who will be included with regs in further analysis) at the time of publication. The 77 articles varied in type between publications. The PB had four reports on research, 21 reports on audit, two surveys and 21 'non-data-containing' articles. the BJP had 14 reports on research, seven case reports, two literature reviews and one 'non-data-containing' article.

A total of 53 replies were received (69\%), 37 from authors in the PB and 16 from the BJP; of these, 34 were SRs and 19 regs. Seventy-two per cent of respondents were male, although the sex distribution of the original sample was unknown. Grade, position of authorship, or type of article were not significantly different between responders and non responders $\left(\chi^{2}\right)$.

SRs took on average 21 months from first involvement to publication and regs 18 months. The time from first involvement to publication differed significantly between journals, averaging 30 months in the BJP and 15 months in the PB. These differences, however, appeared to be accounted for by the differing types of articles in each journal. In the PB, research took an average 30 months from first involvement to publication, audit 11 months, surveys 15 months and 'nondata-containing'articles 12 months. In the BJP, research articles averaged 29 months to publication, case reports 17 months and literature reviews nine months. Research took significantly 
longer to publish compared with other publication types $(P<0.0001$, Mann-Whitney test).

JDs were usually involved from conception $(83 \%)$ to the writing of the finished article $(88 \%)$. Forty-seven per cent worked predominantly in their own time and $28 \%$ did so exclusively. This was remarkably similar between publication types and training grades, with only $11 \%$ reporting carrying out any work while in a research post.

Supervision was usually by a consultant the author had worked for, although $41 \%$ of respondents reported having no supervision from any source. This was less for regs (36\%) than SRs $(44 \%)$ and was accounted for by the different types of publication. Only $12.5 \%$ of authors who published in the BJP reported having no supervision, while $51 \%$ in the PB did so. This striking difference was largely accounted for by the high level of supervision for research $(94 \%)$ and lack of it for published audit $(24 \%)\left(P<0.005, \chi^{2}\right)$.

\section{Need for revisions}

Fifty-nine per cent of papers had to be submitted more than once, and again differed markedly between publications at $49 \%$ in the $P B$ and $87.5 \%$ in the B.JP $\left(P<0.05, \chi^{2}\right)$. Reviewers' comments were generally perceived as helpful and complete revision was rarely required.

\section{Motivation}

Reasons for seeking publication were explored Enhancement of the authors curriculum vitae was unsurprisingly given as a reason for publication by $33(62 \%)$ of respondents, although only one claimed this as the sole motivation. Interest in the subject of publication appeared more important and $46(87 \%)$ gave this as a reason.

\section{Other degrees of qualifications}

Of those who responded, $75 \%$ had two or more previous publications, and this was similar between training grades (13 regs and 27 SRs). Thirteen regs and 24 SRs had other qualifications apart from MBBS and MRCPsych (69\%).

\section{Comment}

This study examined only those who were successful in their quest for publication. It did not examine the characteristics and motivation of those who were unsuccessful in publishing, either through rejection of the article, or fallure to analyse and write up their data. This group may not only differ significantly from those who were unsuccessful, but also from those who successfully publish letters in the PB or BJP, or articles in other journals. The wish of respondents to cast themselves in a positive light and failure to reply by $31 \%$ may have also biased the sample.

Motivation for publication at least in those who successfully publish appears to be primarily interest in the subject, although enhancement of their curriculum vitae also plays a significant role. 'Consultant pressure' appeared a far less important factor, with only $8 \%$ reporting this. Audit and non research articles appear to offer a 'fast track' to publication (being published in a shorter time and with less revisions than research articles); the proportion rejected for publication was not, however, examined. Those seeking publication to enhance their CV should bear in mind that even if their article is accepted it still requires on average one year from first involvement to publication.

While short-listing of candidates on the basis of number of publications is undesirable if clinical and management skills are overlooked, many of the factors associated with publication, such as motivation, perseverance and having an interest in psychiatry that extends beyond 9 to 5 are undoubtedly of legitimate interest to appointment panels.

\section{Acknowledgements}

Thanks to Dista for the financial sponsorship of this study and Professor Greg Wilkinson for comments on the draft of this paper.

\section{References}

IZAGUIRRE. J. (1993) Expectations of senior registrars and those who appoint them. Psychiatric Bulletin. 17, 612-614.

KATONA, C.L.E. \& RoBertSON, M.M. (1993) Who makes it in psychiatry: predictors of success in training grades. Psychiatric Bulletin, 17, 27-29.

LEwIS. S. (1991) The right stufr? A prospective controlled trial of trainees' research. Psychiatric Bulletin, 15, 478480.

Richard Duffett, Registrar, East Ham Memorial Hospital, Shrewsbury Road, London E7 8QR 